\title{
Disclosure of conflicts of interest in biomedical publications
}

\author{
Donald R. Miller MD
}

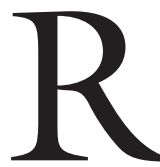

ECENTLY, we provided an update for authors and readers regarding editorial peer review policies of the Journal. ${ }^{1}$ Prior to peer review, manuscripts are screened for compliance with journal policies, including conflict of interest statements, for each submitted manuscript. Despite the fact that issues pertaining to conflict of interest in relation to the sponsorship of clinical trials have been well delineated, ${ }^{2-7}$ we occasionally observe that authors' conflicts of interest statements are incomplete.

Conflicts of interest can exist to varying degrees, and in relation to many different types of publications, including, for example, reports of original investigations of new drugs, or case reports related to new medical devices which the author may have patented. Published letters and certain types of review articles also have the potential for exposure of such conflicts. Incomplete disclosure can be problematic within the context of editorial peer review, and especially, for readers of published manuscripts, for the reasons outlined below.

Simply stated, a conflict of interest exists when "an individual, or organization, is in a position in which professional judgment concerning a primary interest tends to be unduly influenced by a secondary interest, such as financial gain." 8 Secondary gain may involve financial gain, or academic advancement. Financial gain involves direct monetary benefits in the form of stocks or honoraria. In some academic departments, the study findings may affect an author's bonus, or salary (e.g., research related to academic appointments and salary). This too, would be considered a conflict of interest. Having a conflict of interest, in itself, is neither immoral nor unethical, nor would such conflict be grounds for rejection of a manuscript. However, conflicts of interest can influence what is published, and how reports are presented. Furthermore, while a conflict of interest does not, in itself, imply bias, a problem exists whenever one or more conflicts tend to promote bias. ${ }^{9}$ It is for this reason, that transparent and full disclosure is essential.

There are two types of bias for which authors, reviewers, and readers should be vigilant. The first type is publication bias, defined simply as the propensity to report statistically significant results. It is generally recognized that authors are less likely to write reports of negative trials, and journals and reviewers often tend to favour publication of positive reports. While publication bias may exist independently from the source of sponsorship, pharmaceutical companies are more likely to seek publication of results with positive outcomes. In years past, pharmaceuticals sponsored numerous "investigator-driven studies," and statements of competing interests on resulting manuscripts would generally declare that: "This study was supported, in part, by [company ABC]." More recently, with the escalating costs of conducting randomized controlled trials, companies tend to fund large multicentre trials for more definitive answers. For such trials, the sponsor may be involved in the design, data collection, analysis, and writing of related publications. In some cases, the trial sponsor and investigator may be the same, participating trial physicians are often compensated financially, and may even be stockholders of the corporation. Obviously, in such circumstances, the line between sponsors and researchers can easily become blurred, and the need for transparency becomes even greater.

The results of industry-funded trials may also be influenced by inappropriate choice of comparator intervention, or publication bias. There is some evidence that industry-funded trials are more likely to be associated with statistically significant, pro-industry

CAN J ANESTH 2008/ 55: 5 / pp 265-269

From the University of Ottawa, Ottawa, Ontario, Canada.

Address correspondence to: Dr. Donald Miller, Editor-in-Chief, Canadian Journal of Anesthesia, Department of Anesthesia, The Ottawa Hospital, General Campus, CCW 1409, 501 Smyth Road, Ottawa, Ontario K1H 8L6, Canada. Phone: 450-477-7607;

Fax: 450-477-8472; E-mail: cja_office@cas.ca 
findings, both in medical trials and surgical interventions. ${ }^{10}$ In fairness to the pharmaceuticals, some placebo-controlled trials are required for regulatory approval - recognizing that these trials are more likely to generate "positive" findings. Further, the operational quality of multicentre trials sponsored by pharmaceuticals often equals, or exceeds, those funded by other sources.

A second type of bias which tends to be more subtle, and may be difficult to detect, is outcome reporting bias. This is an example of within-study bias, which occurs whenever authors select specific outcomes to include on the report of their research, usually associated with outcomes that appear to reach statistical significance. Studies which have compared study protocols with the resulting full publications have reported a major discrepancy of the primary outcome, in up to $40 \%$ of cases. The problem is that, less effective treatments, and potential adverse events, may be hidden from public view. This may lead to biased estimates of an intervention's effectiveness and safety. This could also result in a skewing of systematic reviews. We have seen examples where sponsorship sources may have generated reporting bias, and the potential impact on validation of clinical practice guidelines should not be underestimated.

It is very difficult for the editor or reviewers to accurately and consistently evaluate the potential for bias in submitted manuscripts, or the degree of sponsor control, without clear conflicts of interest statements which provide full disclosure. Editorial decision making is based upon evaluation of novelty, scientific validity, and overall importance, while there is a trust relationship that declarations of conflicts of interest, of all authors, are presented in a fair and transparent manner. To assist in this process, greater uniformity in policies across journals would be beneficial. The World Association of Medical Editors (www.wame. org/resources/publication-ethics-policies-for-medical-journals), the International Committee of Medical Journal Editors (www.icmje.org) and the Council of Science Editors (www.councilscienceeditors.org/ editorial_policies/white_paper.cfm) now have similar conflict of interest policies. Nevertheless, across biomedical journals, a fairly wide range of conflict of interest policies remains. ${ }^{11}$ For example, some journals may request disclosures, while others require disclosures, which may, or may not, be published. This can generate confusion amongst authors, so that they may not appreciate what they are required to disclose at the time of manuscript submission. On the other hand, we recognize that, not all authors review the Journat's Instructions for Authors prior to submission. Every journal's Instructions for Authors should contain a statement regarding conflict of interest policies. Authors should be aware that such policies are continually evolving, so that journal requirements should be reviewed for updates, prior to each submission. The requirements of the Canadian Journal of Anesthesia (www.cja-jca.org) on this issue are relatively straight forward:

"On the title page, authors must disclose: all [emphasis added] funding sources (departmental, hospital, institutional, commercial, etc.) supporting the submitted work, any commercial or non-commercial affiliations that are, or may be perceived to be a conflict of interest with the work, and any other associations such as consultancies..."

Authors should always err on the side of full disclosure, and whenever there are uncertainties or questions, the editorial office should be contacted. For all manuscripts which are accepted, each author's disclosure of conflict of interest, as well as relevant financial interests and affiliations, and/or declarations of no competing interests, will be published.

To assist in conflict of interest management, we ensure that the policies are made publicly available to the authors, and that disclosures are assessed before the editorial decision is made. Some journals require that all authors sign a statement, separate from the letter of submission, declaring all potential conflicts of interest. We remind authors during the review process about the need to ensure full disclosure, and that disclosure statements will be published. Our policy has evolved, consistent with that of other journals, such that all individuals involved in the review, including board members, guest reviewers, and editors, are also required to declare conflicts of interest relating to the subject matter of the manuscript. As for other aspects of scientific misconduct which may arise during editorial peer review, editors should investigate failures to disclose potential conflicts of interest.

The Journal does not intend to discourage productive working relations between scientific investigators and industry. The complexities of such relationships are not new, and have been well described previously in regards to the anesthesia literature. ${ }^{12}$ At the same time, medical journals are coming under ever-increasing scrutiny in regards to transparency in reporting, and the need to ensure complete documentation of conflicts of interest for all published material is unquestionable. There is potential for considerable bias in scientific publication resulting from conflicts of interest. Contributors are strongly encouraged to review conflicts of interest requirements in the Instructions for Authors, and to be aware that such 
requirements continue to evolve with time. Our readers should expect to be able to formulate their own assessment of the relationship between authors and funding sources, in regards to the conduct and reporting of research, and any other form of peer-reviewed publication. All physicians will wish to have an appreciation of the stakes involved.

\section{La déclaration de con- flits d'intérêt dans les publications biomédi- cales}

Nous avons récemment publié une mise à jour à l'intention de nos auteurs et de nos lecteurs au sujet des politiques du Journal sur la révision éditoriale par

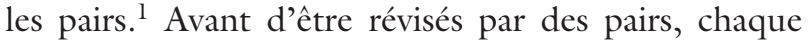
manuscrit est soumis à un contrôle du point de vue de la conformité avec les politiques du Journal, notamment des déclarations de conflits d'intérêt. Malgré le fait que nous ayons clairement défini tous les aspects concernant les conflits d'intérêt par rapport à la commandite d'une étude clinique, ${ }^{2-7}$ il arrive que les auteurs nous fassent parvenir des déclarations de conflits d'intérêt incomplètes.

Les conflits d'intérêt peuvent survenir à différents degrés, et par rapport à de nombreux types de publications, comme par exemple les comptes-rendus de recherches originales sur des nouveaux médicaments, ou des présentations de cas décrivant de nouveaux dispositifs médicaux que l'auteur aurait pu breveter. Les lettres publiées et certains types d'articles de synthèse peuvent également potentiellement exposer de tels conflits. Une déclaration incomplète peut s'avérer problématique dans le contexte de la révision éditoriale par les pairs, et plus particulièrement pour les lecteurs des manuscrits publiés et ce, pour les raisons décrites ci-dessous.

En quelques mots, un conflit d'intérêt existe lorsqu' " une personne ou une organisation se trouve dans une position dans laquelle le jugement professionnel au sujet d'un intérêt primaire peut être excessivement influencé par un intérêt secondaire, comme par exemple le gain financier. ${ }^{8}$ Le gain secondaire peut être financier, mais il peut également être une promotion universitaire. Le gain financier implique des avantages monétaires directs sous forme d'actions ou d'honoraires. Dans certains départements universitaires, les résultats de l'étude pourraient avoir un impact sur le bonus, voire le salaire de l'auteur (par ex. dans le cas de recherches dans le cadre de nominations universitaires ou par rapport au salaire). De tels cas sont également considérés comme provoquant un conflit d'intérêt. Le fait d'avoir un conflit d'intérêt n'est, en soi, ni immoral ni contraire à l'éthique, et un tel conflit ne constituerait pas une raison de rejet de manuscrit. Cependant, les conflits d'intérêt peuvent avoir un impact sur ce qui est publié et sur la manière dont les résultats sont présentés. En outre, bien qu'un conflit d'intérêt n'implique pas forcément un parti pris, un problème survient chaque fois qu'un ou plusieurs conflits tendent à encourager un parti pris. ${ }^{9}$ Pour cette raison, une déclaration transparente et complète est fondamentale.

Il existe deux sortes de parti pris desquels les auteurs, réviseurs et lecteurs doivent se méfier. Premièrement, le biais de publication, qui se définit simplement comme la tendance à rapporter des résultats statistiquement significatifs. Il est généralement admis que les auteurs sont moins enclins à rapporter des résultats d'études négatives, et les revues aussi bien que les réviseurs ont eux aussi tendance à privilégier les résultats positifs. Bien qu'un biais de publication puisse survenir indépendamment de la source de financement, les compagnies pharmaceutiques chercheront vraisemblablement la publication de résultats positifs. Ces dernières années, l'industrie pharmaceutique a commandité nombre d' «études proposées par des chercheurs ", et les déclarations de conflits d'intérêt sur les manuscrits rapportant les résultats de ces études annonçaient généralement que : «Cette étude a été financée, en partie, par [compagnie ABC]». Plus récemment, en raison de l'augmentation rapide des coûts d'études randomisées contrôlées, les compagnies ont tendance à financer d'importantes études multicentriques dans le but d'obtenir des réponses plus définitives. Dans le cadre de telles études, le commanditaire peut avoir son mot à dire dans la conception, la collecte des données, l'analyse et l'écriture des publications associées. Dans certains cas, le commanditaire de l'étude et le chercheur peuvent être la même entité ; les médecins participant à l'étude peuvent être rémunérés et peuvent même être des actionnaires de la corporation. Bien entendu, dans de telles circonstances, la frontière entre commanditaire et chercheur peut aisément devenir floue, et le besoin de transparence est dès lors encore plus important.

Les résultats d'études financées par l'industrie pharmaceutique peuvent également être influencés 
par un choix mal adapté de l'outil de comparaison, ou par un biais de publication. Certaines données probantes démontrent que les études financées par l'industrie pharmaceutique seront plus fréquemment associées à des résultats statistiquement significatifs et pro-industrie, qu'il s'agisse d'études médicales ou d'interventions chirurgicales. ${ }^{10}$ Pour rendre justice aux compagnies pharmaceutiques, certaines études contrôlées par placebo sont nécessaires à une approbation réglementaire, et ainsi ces études engendrent en règle générale des résultats "positifs». De plus, la qualité opérationnelle des études multicentriques financées par des compagnies pharmaceutiques est en générale égale, voire supérieure à celles financées par d'autres sources.

Un deuxième type de parti pris, en général plus subtil et plus difficile à détecter, est le biais dans la présentation des résultats. Il s'agit d'un biais présent au sein même de l'étude, qui survient lorsque les auteurs sélectionnent des résultats spécifiques à inclure dans le compte-rendu de leurs recherches, des données associées en général aux résultats qui semblent atteindre une signification statistique. Les études comparant les protocoles d'études aux publications complètes s'ensuivant ont rapporté une importante divergence du résultat primaire dans jusqu'à $40 \%$ des cas. Le problème réside dans le fait que l'inefficacité d'un traitement et des effets secondaires potentiels sont dissimulés au public. Ceci peut ensuite engendrer des estimations biaisées quant à l'efficacité et l'innocuité d'une intervention donnée. Ceci peut également fausser les comptes-rendus systématiques. Nous avons observé certains cas dans lesquels les sources de commandite pourraient avoir provoqué un biais dans la présentation des résultats, et les répercussions potentielles sur la validation des recommandations de pratique cliniques ne devraient pas être prises à la légère.

Il est extrêmement difficile pour le rédacteur ou pour les réviseurs d'évaluer de manière précise et cohérente le biais potentiel des manuscrits soumis, ou le degré de contrôle exercé par le commanditaire, si les déclarations de conflits d'intérêt ne sont pas claires et ne fournissent pas une divulgation complète. La prise de décision de la rédaction se fonde sur l'évaluation du degré de nouveauté, la validité scientifique, et l'importance globale d'une étude; en outre, une relation de confiance s'établit, selon laquelle les déclarations de conflits d'intérêt de tous les auteurs sont présentées de façon juste et transparente. Une plus grande uniformité dans les politiques des différentes revues encouragerait ce processus. La World Association of Medical Editors (www.wame.org/resources/ publication-ethics-policies-for-medical-journals), l'International Committee of Medical Journal Editors (www.icmje.org) et le Council of Science Editors (www. councilscienceeditors.org/editorial_policies/white_ paper.cfm) ont désormais des politiques semblables en ce qui touche aux conflits d'intérêt. Cependant, il existe encore un éventail de politiques concernant les conflits d'intérêt dans la littérature médicale. ${ }^{11}$ Par exemple, certaines revues demandent des déclarations, alors que d'autres les exigent, déclarations qui pourraient être publiées ou non. Cette situation peut provoquer une certaine confusion chez les auteurs, qui pourrait avoir pour résultat qu'ils ne comprennent pas entièrement ce qu'ils doivent déclarer lors de la soumission de manuscrits. En revanche, nous sommes également conscients que tous les auteurs ne lisent pas forcément les Directives aux Auteurs du Journal avant de soumettre leurs manuscrits. Les Directives aux Auteurs de chaque revue devraient comprendre une section traitant des politiques de déclarations de conflits d'intérêt. De plus, les auteurs devraient être conscients que ces politiques évoluent constamment, et c'est pourquoi les exigences d'une revue devraient être étudiées avant chaque nouvelle soumission. Les exigences du Journal canadien d'anesthésie (www.cjajca.org) concernant cette question sont relativement simples :

"Sur la page de titre, l'auteur devra faire mention de toute (notre emphase) source de financement (service, hôpital, institution, industrie, etc.) du travail présenté. Il faut aussi mentionner, le cas échéant, tout lien commercial qui serait ou pourrait être interprété comme en conflit d'intérêt et de toute autre association, à titre de consultant par exemple... »

Les auteurs devraient toujours tendre vers une déclaration complète ; en cas de doute ou de questions, ils sont invités à contacter la rédaction. Avec chaque manuscrit accepté seront publiés les déclarations de conflits d'intérêt de chaque auteur ainsi que les intérêts financiers et affiliations, et/ou les déclarations de non-conflit d'intérêt.

Afin d'assurer une bonne gestion des conflits d'intérêt, nous nous assurons que les politiques pertinentes sont mises à la disposition des auteurs, et que les déclarations sont évaluées avant la décision de la rédaction. Certaines revues exigent que tous les auteurs signent une déclaration indépendante de la lettre de soumission, dans laquelle sont déclarés tous les conflits d'intérêt potentiels. Pendant le processus de révision, nous rappelons aux auteurs la nécessité d'une déclaration complète ainsi que le fait que toute déclaration sera publiée. Notre politique a évolué en même temps que celle d'autres revues de telle manière 
que toute personne impliquée dans la révision, y compris les membres du conseil, les réviseurs invités et les rédacteurs, doive déclarer quelque conflit d'intérêt que ce soit par rapport au sujet du manuscrit. Concernant d'autres aspects de faute scientifique pouvant survenir durant le processus de révision par les pairs, les rédacteurs ont le devoir d'enquêter sur la non-déclaration de conflits d'intérêt potentiels.

Le Journal n'a pas le dessein de décourager les relations professionnelles productives entre les chercheurs scientifiques et l'industrie pharmaceutique. Le caractère complexe de telles relations n'est pas nouveau, et par le passé ses nombreuses facettes ont été bien décrites en ce qui a trait à la littérature anesthésique. ${ }^{12}$ Dans le même temps, les revues médicales sont de plus en plus passées à la loupe en termes de présentation transparente des résultats. Il est dès lors fondamental que les conflits d'intérêt de tout matériel publié soient documentés de façon précise et exhaustive. Les conflits d'intérêt peuvent provoquer un biais très important dans les publications scientifiques. Nous recommandons vivement aux collaborateurs et auteurs de réviser les exigences concernant les conflits d'intérêt décrites dans les Directives aux Auteurs, et d'être conscients que de telles exigences évoluent sans cesse au fil du temps. Nos lecteurs doivent être en mesure de formuler leur propre jugement concernant la relation entre les auteurs et leurs sources de financement par rapport à l'élaboration et à la présentation des recherches et toute autre forme de publication révisée par les pairs. Tous les médecins souhaiteront avoir une connaissance des enjeux.

\section{References}

1 Miller DR, Donati F. Peer review policies and the Canadian Journal of Anesthesia: An update for authors and readers. Can J Anesth 2007; 54: 1-8.

2 Bekelman JE, Li $\Upsilon$, Gross CP. Scope and impact of financial conflicts of interest in biomedical research: a systematic review. JAMA 2003; 289: 454-65.

3 Boyd EA, Cho MK, Bero LA. Financial conflict-of-interest policies in clinical research: issues for clinical investigators. Acad Med 2003; 78: 769-74.

4 Cho MK, Shohara R, Schissel A, Rennie D. Policies on faculty conflicts of interest at US universities. JAMA 2000; 284: 2203-8.

5 Campbell EG, Gruen RL, Mountford J, Miller LG, Cleary PD, Blumenthal D. A national survey of physician-industry relationships. N Engl J Med 2007; 356: 1742-50.

6 Krimsky S, Rothenberg LS. Financial interest and its disclosure in scientific publications. JAMA 1998; 280: 225-6.
7 DeAngelis CD, Fontanarosa PB, Flanigan A. Reporting financial conflicts of interest and relationships between investigators and research sponsors. JAMA 2001; 286: 89-91

8 Thompson DF. Understanding financial conflicts of interest. N Engl J Med 1993; 329: 573-6.

9 Kassirer JP. On the Take: How Medicine's Complicity with Big Business can Endanger Your Health. NY: Oxford University Press; 2005: 50-1.

10 Bhandari M, Busse JW, Jackowski D, et al. Association between industry funding and statistically significant pro-industry findings in medical and surgical randomized trials. CMAJ 2004; 170: 477-80.

11 Krimsky S, Rothenburg LS. Conflict of interest policies in science and medical journals: editor practices and author disclosure. Sci Eng Ethics 2001; 7: 205-18.

12 Todd MM, Saidman LJ. Academic-industrial relationships: the good, the bad, and the ugly. Anesthesiology. 1997; 87: 197-200. 